\title{
Value of napsin A and thyroid transcription factor-1 in the identification of primary lung adenocarcinoma
}

\author{
PENG ZHANG $^{1}$, YI-PING HAN ${ }^{1}$, LING HUANG ${ }^{2}$, QIANG LI $^{1}$ and DA-LIE MA ${ }^{2}$ \\ Departments of ${ }^{1}$ Respiratory Medicine, and ${ }^{2}$ Pathology, Changhai Hospital, Shanghai 200433, P.R. China
}

Received August 17, 2009; Accepted June 29, 2010

DOI: 10.3892/ol_00000160

\begin{abstract}
Napsin A is a newly discovered functional aspartic proteinase that is expressed in normal lung parenchyma in type II pneumocytes and is thought to be associated with primary lung adenocarcinoma. Thyroid transcription factor- 1 (TTF-1) is a widely used relatively restricted marker for lung adenocarcinoma. The present study aimed to compare the usefulness of napsin A with TTF-1 for the identification of primary lung adenocarcinoma. Immunohistochemical expression of napsin A and TTF-1 was analyzed in 351 lung cancer tissues, including 27 metastases. Napsin A was expressed in 180 of $212(84.9 \%)$ primary lung adenocarcinomas, while no expression was noted in all 27 metastatic lung cancer specimens, including 19 metastatic adenocarcinomas. In contrast, TTF-1 expression was not only noted in 179 of $212(84.4 \%)$ primary lung adenocarcinomas, but also in 12 of 18 (66.7\%) small-cell carcinomas and some of the squamous carcinomas, as well as in one metastatic adenocarcinoma from the thyroid. The sensitivity and specificity of napsin A for primary lung adenocarcinoma (84.9 and $93.8 \%$, respectively) were higher than the sensitivity and specificity of TTF-1 (84.4 and 83.9\%, respectively). By combining napsin A and TTF-1, sensitivity increased to $91.0 \%$. Furthermore, the sensitivity and specificity expression was associated with gender, smoking history, performance status, pathological type, primary tumor size and nodal metastasis. Therefore, napsin A is a useful novel marker in the differential diagnosis of primary lung adenocarcinoma.
\end{abstract}

\section{Introduction}

Lung cancer is the most frequently diagnosed major cancer in the world and the most common cause of cancer-related death, with a yearly growth in incidence (1). By virtue of its abundant blood supply, the lung is also a common site for metastasis from tumors arising at other sites in the body. The therapeutic

Correspondence to: Dr Yi-ping Han, Department of Respiratory Medicine, Changhai Hospital, No. 168 Changhai Road, Shanghai 200433, P.R. China

E-mail: yphan2006@yahoo.com.cn

Key words: napsin A, thyroid transcription factor-1, lung cancer, immunohistochemistry strategies and prognosis differ between different histopathological classifications. However, making a definite diagnosis is not always easy, even for experienced pathologists. In order to improve the diagnostic and prognostic value, molecular markers are needed to facilitate the classification. Napsin A is a recently described aspartic protease, uniquely expressed in lung and kidney cells (2-4). In normal lung it has been found to be present in type II pneumocytes and to be involved in the maturation of the biologically active surfactant protein B (SP-B) (5). Studies have shown its expression in primary lung adenocarcinoma; thus, napsin A can be used in defining primary adenocarcinoma of the lung $(6,7)$. Nevertheless, compared with other markers, there has been limited research involving napsin A. Its usefulness in lung cancer has yet to be clarified.

The present study aimed to examine the prevalence of napsin A in lung cancer tissues, compared with another marker, thyroid transcription factor-1 (TTF-1), which has recently been recognized as a useful marker for lung adenocarcinoma $(8,9)$, and to evaluate their utilization in the identification of primary and metastatic lung cancer. The association of their expression with clinicopathological parameters is also evaluated in this study.

\section{Materials and methods}

Patient selection and tissue sample collection. All patients with lung cancer in this study were from Changhai Hospital, Shanghai, P.R. China, during the period 2007 and 2008. The pathological tissue specimen and clinical data for each patient were colleted prior to treatment. The clinical data included age, gender, smoking history, performance status (PS), histopathological diagnosis, grade of tumor differentiation, tumor stage, primary tumor size and nodal metastasis. Histological subclassification was carried out according to the World Health Organization (WHO) classification. PS was estimated using the Eastern Cooperative Oncology Group (ECOG) scale. Tumor stage was defined according to the International Union Against Cancer (UICC) classification. In total, 324 patients with primary lung cancer and 27 patients with metastatic lung cancer met our study criteria.

Among the 324 patients, 222 cases were male and 102 cases, female (gender ratio, 2.2:1), and the median age was 61 years (range 30-84). A total of 145 patients (44.8\%) had a history of smoking and 264 patients $(81.5 \%)$ had symptoms when first diagnosed, such as cough, expectoration, hemoptysis, chest pain and fever. Adenocarcinoma $(n=212)$ was the most common 
tumor type in the patients $(65.4 \%)$, followed by squamous cell (26.2\%), small-cell (5.6\%), adenosquamous cell (2.5\%) and large-cell carcinoma $(0.3 \%)$. A total of 119 patients $(36.7 \%)$ had stage I-II disease and the remaining 205 patients $(63.2 \%)$ had stage III-IV disease. There were 200 patients $(61.7 \%)$ with nodal metastasis and 284 patients $(87.7 \%)$ with a primary tumor $>3 \mathrm{~cm}$ when diagnosed.

Of the 27 cases with metastatic lung cancer, 8 cases originally developed from mammary adenocarcinoma, 7 from colon carcinoma, 3 from gastric carcinoma, 1 from thyroid adenocarcinoma, 2 from melanoma, 2 from hepatic cellular carcinoma, 2 from uterine cervix cancer and 2 from suprarenal epithelioma.

Immunohistochemistry. All specimens were routinely fixed in $10 \%$ buffered neutral formalin and embedded in paraffin. Each section $(5.0 \mu \mathrm{m})$ was stained with the standard hematoxylin and eosin method for screening review by two experienced pathologists (L.H. and D.L.M.).

Immunohistochemistry was performed using an EnVision two-step method. Primary antibodies included anti-napsin A and anti-TTF-1 antibody (both at a 1:200 dilution, mouse monoclonal antibody; Dako, Denmark). The paraffin was removed from the slides by xylene, and the tissue was rehydrated in various concentrations of ethanol. Hydrogen peroxide (3\%) was added to eliminate endogenous peroxidase activity. The slides were then processed using steam-heat retrieval for $30 \mathrm{~min}$. The antibody was incubated for $30 \mathrm{~min}$ at $37^{\circ} \mathrm{C}$. The abovementioned steps were performed automatically in a Dako automatic immunostainer. Negative controls for napsin A and TTF-1 were carried out by omitting the primary antibody. The positive controls were previously known napsin A- or TTF-1positive lung tissues.

Scoring. For napsin A, only a granular cytoplasmic staining pattern was accepted as positive, while for TTF-1, only a nuclear staining pattern was considered to be positive. The stained slides were observed microscopically by two experienced pathologists. Results were scored in accordance with the criteria of Ueno et al (6) and expressed on a plus-minus scale based on the proportion of tumor cells stained: -, 0-10\%; ,$++ 10-50 \%$ and,$+++ 50-100 \%$.

Statistical analysis. Univariate clinicopathological variables were analyzed using the $\chi^{2}$-test. Variables with $\mathrm{p}<0.05$ in the univariate analysis were included in a Logistic model. The correlation between napsin A and TTF-1 expression was evaluated using Spearman's correlation coefficient. $\mathrm{P}<0.05$ was considered statistically significant. Statistical tests were performed using the software SPSS 13.0 version (SPSS Inc., Chicago, IL, USA).

\section{Results}

Napsin A expression in lung cancer. Immunohistochemistry showed that napsin A was expressed in the cytoplasm with a granular staining pattern, but no staining in the nucleus (Fig. 1). As shown in Table I, napsin A expression was positive in $84.9 \%$ of the lung adenocarcinomas, and $87.5 \%$ in the glandular component of adenosquamous cell carcinomas, but negative in other types of primary lung cancer and metastatic
Table I. Positive expression of napsin A and TTF-1 in lung cancer.

\begin{tabular}{lcccr}
\hline Histology & \multicolumn{2}{c}{ Napsin A } & \multicolumn{2}{c}{ TTF-1 } \\
\hline PLC & $187 / 324$ & $(57.7 \%)$ & $197 / 324$ & $(60.8 \%)$ \\
AdC & $180 / 212$ & $(84.9 \%)$ & $179 / 212$ & $(84.4 \%)$ \\
SCC & $0 / 85$ & $(0.0 \%)$ & $2 / 85$ & $(2.4 \%)$ \\
SCLC & $0 / 18$ & $(0.0 \%)$ & $12 / 18$ & $(66.7 \%)$ \\
ASCC & $7 / 8$ & $(87.5 \%)$ & $4 / 8$ & $(50 \%)$ \\
LCC & $0 / 1$ & $(0.0 \%)$ & $0 / 1$ & $(0.0 \%)$ \\
MLC & $0 / 27$ & $(0.0 \%)$ & $1 \mathrm{a} / 27$ & $(3.7 \%)$ \\
\hline
\end{tabular}

PLC, primary lung cancer; MLC, metastatic lung cancer; AdC, adenocarcinoma; SCC, squamous cell carcinoma; SCLC, small-cell lung carcinoma; ASCC, adenosquamous cell carcinoma; LCC, largecell carcinoma. ${ }^{\mathrm{a} T h e}$ TTF-1-positive metastatic lung cancer is from thyroid adenocarcinoma.

lung cancer cases. Napsin A had a high specificity of $93.8 \%$ to lung adenocarcinomas. Comparatively, napsin A expression was also noted in normal type II pneumocytes and some alveolar macrophages near the neoplasms, while no expression was observed in type I pneumocytes, bronchiolar epithelium, bronchial epithelium and stromal cells.

TTF-1 expression in lung cancer. TTF-1 expression was located in the nucleus with no staining on the membrane and in the cytoplasm (Fig. 2). A positive expression was observed mostly in adenocarcinomas, small-cell carcinomas and the glandular component of adenosquamous cell carcinomas. However, TTF-1 expression was negative in the squamous component of adenosquamous cell carcinoma, large-cell carcinoma and most of the squamous cell carcinoma specimens. Its sensitivity and specificity to adenocarcinoma were 84.4 and $83.9 \%$, respectively. Nevertheless, positive nuclear staining was also observed in normal pulmonary alveolar pneumocytes near the neoplastic cells, but it was not noted in the stromal and infiltrating inflammatory cells. The metastatic lung cancer specimens were TTF-1-negative except for one adenocarcinoma from the thyroid.

Association of TTF-1 and napsin A expression with clinicopathological parameters in primary lung cancer. The expression of napsin A and TTF-1 increased significantly in female and male patients with a history of smoking $(\mathrm{p}<0.001)$. Their positive expression was also associated with patients without symptoms (PS score, $0 ; \mathrm{p}<0.01$ ). In lung adenocarcinoma, both napsin A and TTF-1 expression was positively related to differentiation $(\mathrm{p}<0.001)$, since poorly differentiated adenocarcinoma had a lower expression. An increased expression of both napsin A and TTF-1 was noted in patients with a primary tumor size $<3 \mathrm{~cm}(\mathrm{p}<0.01)$ and in those without nodal metastasis $(\mathrm{p}<0.05)$. No significant relationships were observed between napsin A and TTF-1 expression and age and TNM stage (Table II).

Logistic multivariate analysis demonstrated that napsin A and TTF-1 expression was associated with smoking history $(\mathrm{p}<0.01)$ 
Table II. Expression of napsin A and TTF-1 in NSCLC and their association with clinicopathological variables.

\begin{tabular}{|c|c|c|c|c|c|c|c|c|}
\hline & \multicolumn{4}{|c|}{ Napsin A } & \multicolumn{4}{|c|}{ TTF-1 } \\
\hline & + & - & $\begin{array}{l}\text { Positive } \\
\text { rate }(\%)\end{array}$ & P-value & + & - & $\begin{array}{l}\text { Positive } \\
\text { rate }(\%)\end{array}$ & P-value \\
\hline \multicolumn{9}{|l|}{ Age } \\
\hline$<61$ & 93 & 54 & 63.3 & & 90 & 57 & 61.2 & \\
\hline$\geq 61$ & 94 & 83 & 53.1 & 0.065 & 107 & 70 & 60.5 & 0.887 \\
\hline \multicolumn{9}{|l|}{ Gender } \\
\hline Male & 107 & 115 & 48.1 & & 117 & 105 & 52.7 & \\
\hline Female & 80 & 22 & 78.4 & $<0.001$ & 80 & 22 & 78.4 & $<0.001$ \\
\hline \multicolumn{9}{|l|}{ Smoking } \\
\hline Never & 113 & 32 & 77.9 & & 114 & 31 & 78.6 & \\
\hline Ever & 74 & 105 & 41.3 & $<0.001$ & 83 & 96 & 48.4 & $<0.001$ \\
\hline \multicolumn{9}{|c|}{ Performance status } \\
\hline 0 & 46 & 14 & 76.7 & & 47 & 13 & 78.3 & \\
\hline 1,2 & 141 & 123 & 53.4 & $<0.001$ & 150 & 114 & 56.8 & 0.002 \\
\hline \multicolumn{9}{|c|}{ Differentiation $^{\mathrm{a}}$} \\
\hline Well & 173 & 18 & 90.6 & & 174 & 17 & 91.1 & \\
\hline Poor & 7 & 14 & 33.3 & $<0.001$ & 6 & 15 & 28.6 & $<0.001$ \\
\hline \multicolumn{9}{|c|}{ Tumor size } \\
\hline$\leq 3 \mathrm{~cm}$ & 31 & 9 & 77.5 & & 35 & 5 & 87.5 & \\
\hline$>3 \mathrm{~cm}$ & 156 & 128 & 54.9 & 0.007 & 162 & 122 & 57.0 & $<0.001$ \\
\hline \multicolumn{9}{|c|}{ Nodal metastasis } \\
\hline No & 85 & 39 & 68.5 & & 84 & 40 & 67.7 & \\
\hline Yes & 102 & 98 & 51.0 & 0.002 & 113 & 87 & 56.5 & 0.044 \\
\hline \multicolumn{9}{|l|}{ TNM } \\
\hline I-II & 74 & 45 & 62.2 & & 74 & 45 & 62.2 & \\
\hline III-IV & 113 & 92 & 55.1 & 0.215 & 123 & 82 & 60.0 & 0.698 \\
\hline \multicolumn{9}{|l|}{ TTF-1 } \\
\hline+ & 166 & 13 & & & & & & \\
\hline- & 14 & 19 & & $<0.001$ & & & & \\
\hline
\end{tabular}

aIn lung adenocarcinoma, 'well' indicates well or moderately differentiated; 'poor' indicates poorly differentiated tumors. NSCLC, non-small cell lung carcinoma.

Table III. Logistic regression analysis of napsin A and TTF-1 expression in lung cancer.

\begin{tabular}{lccrr}
\hline & \multicolumn{2}{c}{ Napsin A } & & TTF-1 \\
\cline { 2 - 5 } & B & Significance & B & Significance \\
\cline { 3 - 5 } Gender & 0.836 & 0.026 & 0.640 & 0.060 \\
Smoking history & -0.972 & 0.003 & -0.976 & 0.002 \\
Histology & -2.228 & 0.000 & -1.354 & 0.000 \\
Tumor size & -0.927 & 0.104 & 0.011 & 0.946 \\
Nodal metastasis & -0.216 & 0.493 & 0.086 & 0.544 \\
Performance status & -0.372 & 0.382 & -0.855 & 0.020 \\
Constant & 6.017 & 0.000 & 3.449 & 0.000 \\
\hline
\end{tabular}

and pathological type $(\mathrm{p}<0.001)$. Expression rates were higher in patients with adenocarcinoma or an absence of smoking history (Table III). Napsin A was correlated with gender $(\mathrm{p}=0.026)$ and TTF-1 with PS $(\mathrm{p}=0.020)$.
Correlation of napsin A and TTF-1 expression in lung adenocarcinoma. Table IV shows the phenotype of lung adenocarcinoma by immunohistochemical staining. Combining napsin A and TTF-1 resulted in sensitivity to lung adenocar- 
Table IV. Napsin A and TTF-1 expression in primary lung adenocarcinoma.

\begin{tabular}{lccrr}
\hline & $\mathrm{Napsin}^{+} / \mathrm{TTF}^{+}$ & $\mathrm{Napsin}^{+} / \mathrm{TTF}^{-}$ & $\mathrm{Napsin}^{-} / \mathrm{TTF}^{+}$ & $\mathrm{Napsin}^{-} / \mathrm{TTF}^{-}$ \\
\hline Adenocarcinoma & 166 & 14 & 13 & 19 \\
Well or moderately differentiated & 163 & 10 & 10 & 8 \\
Poorly differentiated & 3 & 4 & 3 & 11 \\
\hline
\end{tabular}

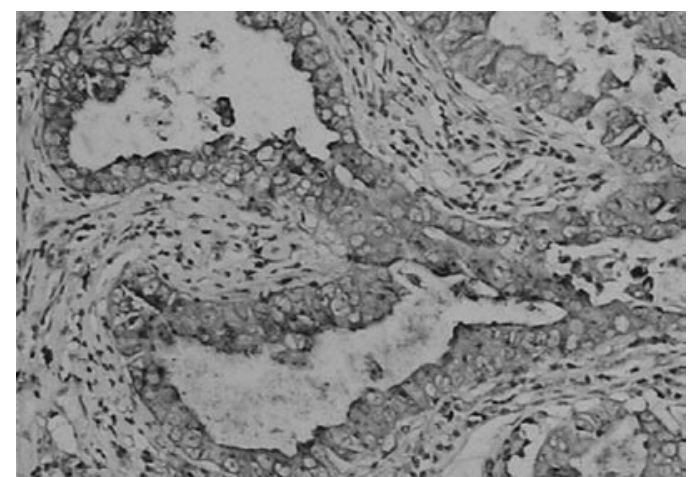

Figure 1. Napsin A is expressed in the cytoplasm of primary lung adenocarcinoma with a granular staining pattern (EnVision two-step method; magnification, $\mathrm{x} 200$ ).

cinoma increasing to $91.0 \%$ (193/212), whereas the specificity was $80.4 \%$ (90/112). Using Spearman's correlation coefficient analysis, the expression of napsin A significantly correlated with TTF-1 in lung adenocarcinomas $(\mathrm{r}=0.510, \mathrm{p}<0.001)$, as well as in moderately and well-differentiated adenocarcinomas $(r=0.387, p<0.001)$. Napsin A expression also positively correlated with TTF-1 in poorly differentiated adenocarcinomas, but without significance $(r=0.224, p=0.330)$.

\section{Discussion}

Napsin A is a new member of the aspartic protease family, with a molecular weight of $35 \mathrm{kDa}$ and an isoelectric point of 5.29. In 1998, Tatnell et al (2) reported the naspin A gene for the first time. Prior to this study, a pair of polypeptide markers for lung adenocarcinoma, TAO1/TAO2, were described using two-dimensional gel electrophoresis $(10,11)$. TAO2 was later demonstrated to be identical to napsin A $(3,12)$. In our study, napsin A was expressed in $84.9 \%$ of the primary lung adenocarcinomas with a granular cytoplasmic pattern. No positive expression was noted in squamous cell carcinoma, small-cell or large-cell carcinomas. Napsin A had a high specificity of $93.8 \%$ to lung adenocarcinoma. In the immunohistochemical staining, napsin A-positive cells were also observed in the glandular component of the adenosquamous cell carcinoma specimens, while cells in the squamous component were all negative. To the best of our knowledge, napsin A expression in adenosquamous cell carcinoma has never been examined. When analyzed with clinical data, napsin A was observed more frequently in female non-smokers who are susceptible to lung adenocarcinoma. We also studied napsin A expression in 27 metastatic lung cancer cases, including 19 metastatic lung adenocarcinomas. They

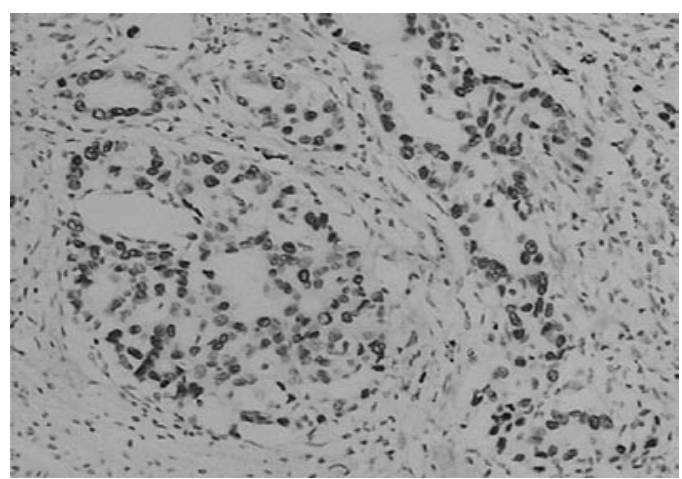

Figure 2. TTF-1 is expressed in the nucleus of primary lung adenocarcinoma (EnVision two-step method; magnification, x200).

were all negative for napsin A staining. The above observation further demonstrated the tissue-specific expression of napsin A in lung adenocarcinoma. By virtue of its specificity in lung adenocarcinoma, studies have focused on its value in the diagnosis of primary and metastatic adenocarcinoma of the lung. Ueno et al (6) examined the expression of napsin in 118 lung tissues, including 16 metastases by in situ hybridization. Napsin was expressed in the tumor cell compartment in 33 of 39 lung adenocarcinomas (84.6\%). Only one metastasis of a renal carcinoma origin expressed napsin, while none of the other types of tumors expressed napsin. Suzuki et al (13) showed that napsin A was expressed in almost all tumor cells in most primary lung adenocarcinoma specimens $(84.3 \%, 70 / 83)$. Its expression was not observed in any of the 32 metastatic lung tumors (colon, stomach, breast, uterus, thyroid and submandibular gland), nor in any of the primary sites of adenocarcinoma of other organs (stomach, colon, breast and thyroid). These results are largely in accordane with our observations.

This study analyzed the association of napsin A with clinicopathological parameters. The expression of napsin A was significantly higher in patients without nodal metastasis or symptoms, or with a primary tumor size $<3 \mathrm{~cm}$, compared with their counterparts. It was also found to be associated with a high degree of differentiation in adenocarcinoma. These findings indicate napsin A may be important in the carcinogenesis of lung cancer. A number of aspartic proteinases are reported to be involved in cancer progression. However, few studies exist on the role of napsin A. Nevertheless, napsin A was suggested to be involved in the processing of proSP-B in type II pneumocytes in the human lung. Knockdown of napsin A by small interfering RNA resulted in decreased levels of mature SP-B (14). In this context, napsin A is a significant enzyme 
in type II pneumocytes. In a recent study (15), napsin A was re-expressed in the tumorigenic HEK 293 kidney cell line. Cells expressing napsin A showed a reduced capacity for anchorage-independent growth, and formed tumors in SCID mice with a lower efficiency and slower onset compared to vector-transfected control cells. On the other hand, napsin A staining was associated with tumor differentiation. Taken together, these observations suggest the crucial role napsin A plays in lung cancer. The loss of napsin A expression may enhance the aggressive behavior of lung cancer, influencing disease prognosis. Napsin A may also be related to patient prognosis in a yet unidentified manner.

Furthermore, the expression of napsin A was significantly correlated with TTF-1 in lung adenocarcinoma. TTF-1 was identified as an independent prognostic factor for survival, besides PS and TNM stage $(16,17)$. In the meta-analysis by Berghmans et al (18), TTF-1 positivity was associated with a more favorable survival in non-small cell lung cancer, mainly in early and locally advanced stages, and adenocarcinoma. Our study showed that TTF-1, as well as napsin A, exhibit a higher expression in patients with a PS of 0 . Based on this observation, we hypothesize that napsin A is also a prognostic factor in lung cancer, although further investigations are needed to better define its prognostic role in this malignancy.

In the 324 primary lung cancer samples, napsin A and TTF-1 had a similar sensitivity, while napsin A had a much higher specificity to lung adenocarcinoma compared with TTF-1 (93.8 vs. 83.9\%). Napsin A expression was almost limited to adenocarcinoma, while TTF-1 was not so specific. Although TTF-1 was expressed in $84.4 \%$ of the adenocarcinoma specimens, its expression was also noted in a large proportion of small-cell lung carcinomas, as well as some of the squamous cell carcinomas, in accordance with previous studies $(19,20)$. Furthermore, by virtue of its tissue-specific exclusive expression in thyroid neoplasms, it would be difficult to distinguish primary lung adenocarcinoma from metastatic lung adenocarcinoma from the thyroid based on clinical and morphological features. In contrast, napsin A is much more specific to lung adenocarcinoma. Although certain renal cell carcinomas have been reported to express napsin A, it is thought that these false positives are likely due to the presence of intrinsic biotin, which is readily detected on negative controls (8). Subsequently, napsin A may be an alternative to TTF-1 for the identification of primary adenocarcinoma of the lung. False negatives exist in lung adenocarcinomas when using either napsin A or TTF-1, but these may be reduced by using the two markers together. In our clinical practice, the combined use of napsin A and TTF-1, as well as morphology and clinical information, may facilitate the diagnosis and differentiation of lung cancer.

In conclusion, napsin A has a tissue-specific expression in primary lung adenocarcinoma and may be involved in the process of carcinogenesis. Napsin A compared with TTF-1 may be more useful in the identification of primary lung adenocarcinoma.

\section{References}

1. Weir HK, Thun MJ, Hankey BF, et al: Annual report to the nation on the status of cancer, 1975-2000, featuring the uses of surveillance data for cancer prevention and control. J Natl Cancer Inst 95: 1276-1299, 1998.

2. Tatnell PJ, Powell DJ, Hill J, et al: Napsins: new human aspartic proteinases. Distinction between two closely related genes. FEBS Lett 441: 43-48, 1998.

3. Chuman Y, Bergman A, Ueno T, et al: Napsin A, a member of the aspartic protease family, is abundantly expressed in normal lung and kidney tissue and is expressed in lung adenocarcinomas. FEBS Lett 462: 129-134, 1999.

4. Schauer-Vukasinovic V, Bur D, Kling D, et al: Human napsin A: expression, immunochemical detection and tissue localization. FEBS Lett 462: 135-139, 1999.

5. Brasch F, Ochs M, Kahne T, et al: Involvement of napsin A in the $\mathrm{C}$ - and $\mathrm{N}$-terminal processing of surfactant protein B in type-II pneumocytes of the human lung. J Biol Chem 278: 49006-49014, 2003.

6. Ueno T, Linder S and Elmberger G: Aspartic proteinase napsin is a useful marker for diagnosis of primary lung adenocarcinoma. Br J Cancer 88: 1229-1233, 2003.

7. Hirano T, Gong Y, Yoshida K, et al: Usefulness of TA02 (napsin A) to distinguish primary lung adenocarcinoma from metastatic lung adenocarcinoma. Lung Cancer 41: 155-162, 2003.

8. Jagirdar J: Application of immunohistochemistry to the diagnosis of primary and metastatic carcinoma to the lung. Arch Pathol Lab Med 132: 384-396, 2008.

9. Chang YL, Lee YC, Liao WY, et al: The utility and limitation of thyroid transcription factor-1 protein in primary and metastatic pulmonary neoplasms. Lung Cancer 44: 149-157, 2004.

10. Hirano T, Franzén B, Uryu K, et al: Detection of polypeptides associated with the histopathological differentiation of primary lung carcinoma. Br J Cancer 72: 840-848, 1995.

11. Hirano T, Fujioka K, Franzén B, et al: Relationship between TA01 and TA02 polypeptides associated with lung adenocarcinoma and histocytological features. Br J Cancer 75: 978-985, 1997.

12. Hirano T, Auer G, Maeda M, et al: Human tissue distribution of TA02, which is homologous with a new type of aspartic proteinase, napsin A. Jpn J Cancer Res 91: 1015-1021, 2000.

13. Suzuki A, Shijubo N, Yamada G, et al: Napsin A is useful to distinguish primary lung adenocarcinoma from adenocarcinomas of other organs. Pathol Res Pract 201: 579-586, 2005.

14. Ueno T, Linder S, Na CL, et al: Processing of pulmonary surfactant protein B by napsin and cathepsin H. J Biol Chem 279: 16178-16184, 2004.

15. Ueno T, Elmberger G, Weaver TE, et al: The aspartic protease napsin A suppresses tumor growth independent of its catalytic activity. Lab Invest 88: 256-263, 2008.

16. Bruno MD, Bohinski RJ, Huelsman KM, et al: Lung cell-specific expression of the murine surfactant protein $\mathrm{A}$ (SP-A) gene is mediated by interactions between the SP-A promoter and thyroid transcription factor-1. J Biol Chem 270: 6531-6536, 1995.

17. Barlési F, Pinot D, Legoffic A, et al: Positive thyroid transcription factor 1 staining strongly correlates with survival of patients with adenocarcinoma of the lung. Br J Cancer 93: 450-452, 2005.

18. Berghmans T, Paesmans M, Mascaux C, et al: Thyroid transcription factor 1 - a new prognostic factor in lung cancer: a meta-analysis. Ann Oncol 17: 1673-1676, 2006.

19. Tan D, Li Q, Deeb G, et al: Thyroid transcription factor-1 expression prevalence and its clinical implications in non-small cell lung cancer: a high-throughput tissue microarray and immunohistochemistry study. Hum Pathol 34: 597-604, 2003.

20. Kaufmann O and Dietel M: Expression of thyroid transcription factor-1 in pulmonary and extrapulmonary small cell carcinomas and other neuroendocrine carcinomas of various primary sites. Histopathology 36: 415-420, 2000. 